\title{
Dietary intakes among South Asian adults differ by length of residence in the USA
}

\author{
Sameera A Talegawkar ${ }^{1} *$, Namratha R Kandula ${ }^{2}$, Meghana D Gadgil ${ }^{3}$, Dipika Desai ${ }^{4}$ \\ and Alka M Kanaya ${ }^{3}$ \\ ${ }^{1}$ Center for Human Nutrition, Department of International Health, Johns Hopkins Bloomberg School of Public Health, \\ 615 N. Wolfe Street, Room W2041, Baltimore, MD 21205, USA: ²Division of General Internal Medicine, \\ Northwestern University, Chicago, IL, USA: ${ }^{3}$ Division of General Internal Medicine, University of California, \\ San Francisco, San Francisco, CA, USA: ${ }^{4}$ Population Health Research Institute, Hamilton Health Sciences, Hamilton, \\ Ontario, Canada
}

Submitted 9 October 2014: Final revision received 16 March 2015: Accepted 10 April 2015: First published online 20 May 2015

\begin{abstract}
Objective: To examine whether nutrient and food intakes among South Asian adult immigrants differ by length of residence in the USA.

Design: Cross-sectional analysis to examine differences in nutrient and food intakes by length of residence in the USA. Dietary data were collected using an interviewer-administered, culturally appropriate FFQ, while self-reported length of residence was assessed using a questionnaire and modelled as tertiles.

Setting: The Mediators of Atherosclerosis in South Asians Living in America (MASALA) study.

Subjects: Eight hundred and seventy-four South Asians (mean age $=55$ (SD 9) years; $47 \%$ women; range of length of residence in the USA $=2-58$ years), part of the baseline examination of the MASALA study.

Results: Intakes of fat, including saturated and trans fats, dietary cholesterol and $n$ - 6 fatty acids, were directly associated with length of residence, while intakes of energy, carbohydrate, glycaemic index and load, protein, dietary fibre, folate and $\mathrm{K}$ were inversely associated with length of residence $(P$ trend $<0.05)$. A longer length of residence in the USA was also associated with higher intakes of alcoholic beverages, mixed dishes including pizza and pasta, fats and oils, and lower intakes of beans and lentils, breads, grains and flour products, milk and dairy products, rice, starchy vegetables and sugar, candy and jam ( $P$ for differences across groups $<0 \cdot 05)$.

Conclusions: Length of residence in the USA influences diet and nutrient intakes among South Asian adult immigrants and should be considered when investigating and planning dietary interventions to mitigate chronic disease risk.
\end{abstract}

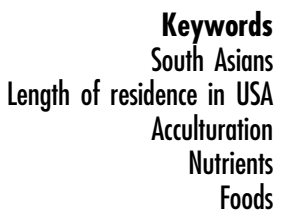

Keywords

ence in USA

Nutrients

Foods
The 2010 US census showed that between 2000 and 2010, the US Asian population grew faster than any other race in the country ${ }^{(1)}$. At 3.4 million, South Asians, those who trace their origins to countries such as India, Pakistan, Bangladesh, Nepal and Sri Lanka, are among the second fastest-growing ethnic community in the country ${ }^{(1)}$. There is some evidence to suggest that South Asians in the USA bear a disproportionate burden of chronic disease including type 2 diabetes and heart disease, some of it attributable to the adoption of Western-style dietary patterns and a sedentary lifestyle ${ }^{(2-4)}$.

Acculturation, a process in which members of one cultural group adopt the beliefs and behaviours of another group, is a complex, multidimensional and dynamic process $^{(5,6)}$ and studies of US immigrants have shown mixed consequences of immigration and acculturation on diet and health outcomes. For example, some studies with Hispanics have demonstrated the replacement of healthful dietary patterns and practices with those emphasizing processed and fast-food type foods higher in saturated fats and added sugars ${ }^{(7,8)}$, and lower in fruit and vegetables ${ }^{(9,10)}$, whole grains and dietary fibre ${ }^{(11)}$. Others have shown positive consequences with higher consumption of low-fat meats and fish ${ }^{(12)}$, cereal fibre ${ }^{(12,13)}$, fruit ${ }^{(14)}$, nonstarchy vegetables and lower-glycaemic-index foods ${ }^{(13)}$.

A recent review of studies examining the changes in dietary habits of South Asians after migration to European 
countries reported increases in energy and fat intakes, decreases in the consumption of total carbohydrates with an increased emphasis on refined sources, higher intakes of meat and dairy products, and a reduction in the consumption of fruit and vegetables resulting in an overall decrease in intakes of dietary fibre, conferring a higher risk of chronic disease ${ }^{(15)}$.

Detailed investigations examining the role of immigration and acculturation on dietary intakes are lacking for South Asian populations residing in the USA. We therefore examined differences in nutrient and food intakes across South Asian immigrants classified on the basis of length of residence in the USA, which we used as a proxy for acculturation status. We hypothesized that nutrient and food intakes would differ by length of residence in the USA. Specifically, a longer length of residence in the USA would be associated with consumption of a less traditional diet with lower intakes of beans and lentils, breads, grain and flour preparations, and rice, and higher intakes of alcoholic beverages and animal products, leading to higher intakes of fat, protein and dietary cholesterol and lower intakes of carbohydrate and dietary fibre as compared with those having a shorter length of residence in the USA.

\section{Methods}

\section{Participants and study design}

The present analyses are based on data collected for the baseline examination of the Mediators of Atherosclerosis in South Asians Living in America (MASALA) study. The overall goals of the MASALA study are to study the prevalence, correlates and outcomes associated with subclinical atherosclerosis in a community-based sample of South Asian men and women between the ages of 40 and 84 years from two field centres. A detailed description of the study rationale, design, as well as methods has been provided elsewhere ${ }^{(16)}$. Briefly, using a community-based sample, 906 South Asians (mean age $=55$ (SD 9) years; $46 \%$ women; $98 \%$ immigrants who have lived for 27 (SD 11) years in the USA, range $=2-58$ years) were recruited from the two clinical sites including the nine counties of the San Francisco Bay area (representing the University of California at San Francisco (UCSF) field site) and seven census tracts closest to the Northwestern University (NWU) medical centre, as well as suburban locations with high proportions of South Asian residents as suggested by census data. The overall enrolment rate was $60 \cdot 8 \%$ ( $52 \%$ at UCSF and $77 \%$ at NWU).

Data collection was performed at the clinical sites and relevant to this analysis included: (i) administration of questionnaires querying demographic information, tobacco use, alcohol consumption, medical conditions, physical activity and dietary intakes; and (ii) a physical examination including anthropometry. The study was conducted according to the guidelines laid down in the Declaration of Helsinki and all procedures involving human subjects were approved by the institutional review boards of UCSF and NWU. All study participants signed informed consent. Further, the institutional review board of Johns Hopkins Bloomberg School of Public Health deemed this secondary analysis plan using de-identified data as not constituting human subjects research.

\section{FFQ used in the MASALA study}

Dietary intakes in the MASALA study were assessed using the interviewer-administered Study of Health Assessment and Risk in Ethnic groups (SHARE) FFQ. The SHARE FFQ was developed for use among South Asians residing in Canada and its relative validity has been previously documented $^{(17)}$. The FFQ includes 163 items with openended frequency responses and three serving sizes for each food item. The quantity for a medium (or average) serving size was indicated on the form, small was 0.5 or less than medium and large was 1.5 or more than medium. The ESHA Food Processor nutrient analysis software version 6.11 (1996) was used to derive energy and nutrient estimates.

Energy, macro- and micronutrients, and self-reported intakes of foods and food groups, expressed as number of medium servings per week (Appendix) derived from the FFQ, were examined.

Prior to analysis, as has been done previously ${ }^{(18,19)}$, participants ( $n$ 13) with implausible dietary intake information (defined as $\leq 3347$ or $\geq 16736 \mathrm{~kJ} / \mathrm{d}$ for men and $\leq 2092$ or $\geq 14644 \mathrm{~kJ} / \mathrm{d}$ for women) were excluded.

\section{Length of residence in the USA}

Length of residence in the USA was derived by a question asking the study participants about this. For analysis purposes, length of residence in the USA was categorized into tertiles in the cohort. Information for this variable was available for 887 of the MASALA study participants.

\section{Statistical analyses}

Differences in sociodemographic and anthropometric variables across tertiles of length of residence in the USA were examined. For variables expressed on a continuous scale, differences were examined using $t$ tests; for categorical variables, homogeneity across strata was tested with the $\chi^{2}$ test. All nutrients except glycaemic index and those expressed as percentages of total energy intake were log-transformed prior to analysis; however, for clarity and greater interpretability, we present adjusted means for untransformed nutrients. Based on results from univariate analysis, final models were adjusted for age, sex, education and daily energy intake. Models were adjusted for multiple comparisons using Dunnett's adjustment. Tests for trend were performed by assigning the median value of the length of residence for each tertile and treating these as a continuous variable, adjusting for all covariates. 


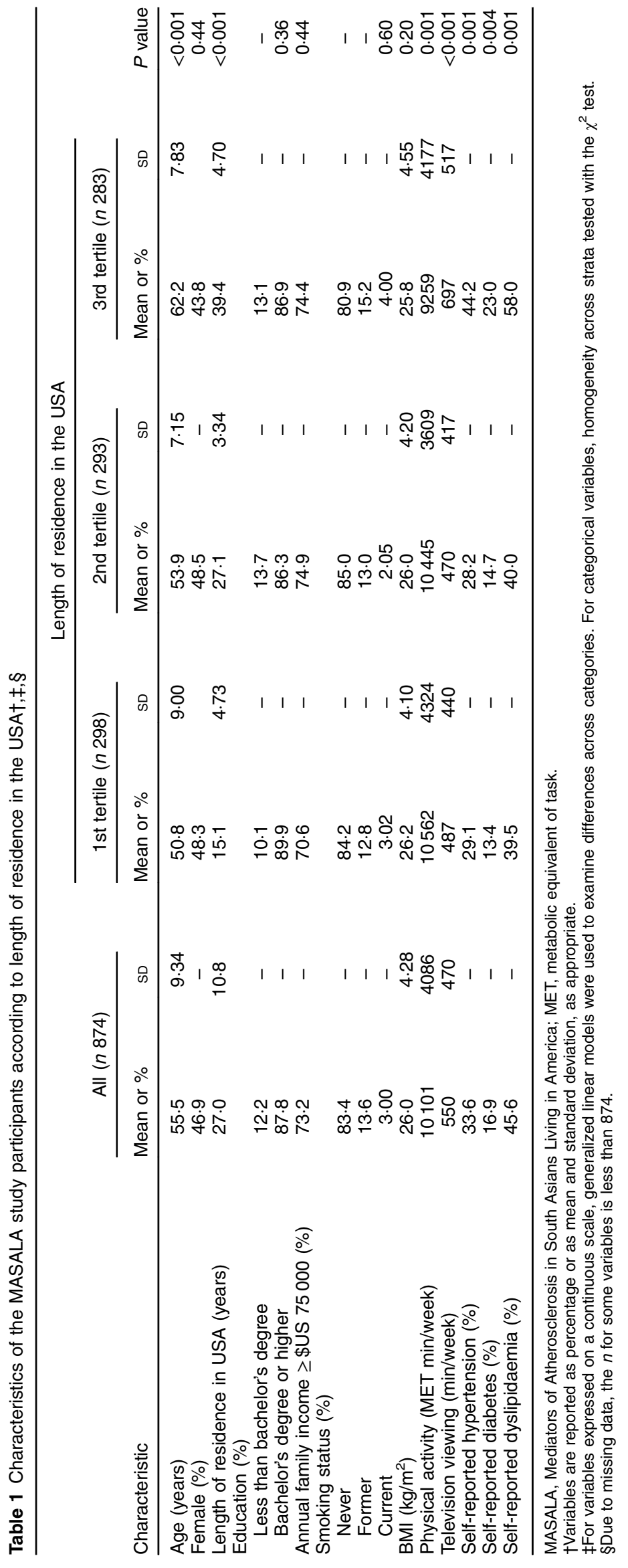


In order to determine whether there was effect modification by sex, age and education, we included interaction terms of them. Because we were testing multiple interactions, to avoid false positives, only those that were significant at $P<0.01$ were examined further. None of these terms were statistically significant $(P>0 \cdot 01)$ and hence were dropped from the models. Intakes of foods and food groups (servings/week) were non-normally distributed and differences between groups were tested using the Kruskal-Wallis test. Analyses were performed using the statistical software package SAS version $9 \cdot 3$ and all statistical tests were two-sided with significance level set at $P<0 \cdot 05$.

\section{Results}

The characteristics of the study population are shown in Table 1. The mean age was 55.5 (SD 9.34) years, with women constituting $47 \%$ of the cohort. Length of residence in the USA was directly associated with age $(P<0.001)$. More than $87 \%$ had at least a bachelor's degree and over $73 \%$ reported an annual family income above \$US 75000 . The mean BMI was $26 \cdot 0$ (SD $4 \cdot 28$ ) $\mathrm{kg} / \mathrm{m}^{2}$ and the sample was constituted predominantly of never smokers ( $83 \%$ ). No differences in education, income, sex distribution, BMI or smoking status were observed with respect to length of residence in the USA. Weekly physical activity was inversely associated and television viewing was directly associated with length of residence in the USA. Similarly, prevalence of self-reported of chronic diseases, including hypertension, type 2 diabetes and dyslipidaemia, was directly associated with length of residence in the USA.
Intakes of energy, carbohydrate (total and as a percentage of energy), glycaemic index and load, protein (total and as a percentage of energy), dietary fibre, folate and $\mathrm{K}$ were inversely associated with length of residence in the USA; while intakes of fat (total and as a percentage of energy), saturated and trans fat, dietary cholesterol and $n$ - 6 fatty acids were directly associated with length of residence in the USA (Tables 2 and 3).

Intakes of alcoholic beverages, fats and oil, and mixed dishes differed across the tertiles of length of residence in the USA and were higher in the highest tertile compared with the lowest and/or the middle tertile; whereas intakes of beans and lentils, breads, cereals and grains, rice and rice preparations, sugar, candy and jam, and starchy vegetables were higher in the lowest and/or middle tertile compared with the highest tertile of length of residence in the USA (Table 4).

\section{Discussion}

The objective of the present study was to evaluate whether dietary and nutrient intakes differed by length of residence in the USA in a cohort of middle-aged and older South Asians. In general, a longer length of residence in the USA was associated with lower daily intakes of energy, carbohydrate, dietary fibre, glycaemic index and load, and higher intakes of fat, protein and dietary cholesterol. When weekly consumption of foods and food groups was examined, a longer length of residence in the USA was associated with higher intakes of alcoholic beverages and mixed dishes, fats and oil, and lower intakes of beans and

Table 2 Daily energy and macronutrient intakes of the MASALA study participants according to length of residence in the USA†

\begin{tabular}{|c|c|c|c|c|c|c|c|}
\hline \multirow[b]{3}{*}{ Nutrient (/d) } & \multicolumn{6}{|c|}{ Length of residence in the USA } & \multirow[b]{3}{*}{$P$ for trend } \\
\hline & \multicolumn{2}{|c|}{ 1st tertile (n 298) } & \multicolumn{2}{|c|}{ 2nd tertile (n 293) } & \multicolumn{2}{|c|}{ 3rd tertile (n 283) } & \\
\hline & Mean & SE & Mean & SE & Mean & SE & \\
\hline Energy (kJ) & $7373^{\star}$ & 126 & 7017 & 120 & 6734 & 135 & 0.0004 \\
\hline Carbohydrate $(\mathrm{g})$ & $251^{*}$ & 1.48 & $248^{*}$ & 1.42 & 240 & 1.59 & 0.0001 \\
\hline Carbohydrate (\% of energy) & $57 \cdot 4^{\star}$ & 0.35 & $56 \cdot 7$ & 0.33 & $55 \cdot 0$ & 0.37 & $<0.0001$ \\
\hline Protein $(g)$ & $62 \cdot 6^{*}$ & 0.57 & $61 \cdot 8$ & 0.54 & $61 \cdot 0$ & 0.61 & 0.04 \\
\hline Protein (\% of energy) & $14 \cdot 9^{\star}$ & 0.13 & $14 \cdot 6$ & 0.12 & 14.4 & 0.14 & 0.02 \\
\hline Total fat $(\mathrm{g})$ & $53 \cdot 1^{*}$ & 0.55 & $54 \cdot 6^{*}$ & 0.53 & $56 \cdot 7$ & 0.59 & 0.0003 \\
\hline Saturated fat $(\mathrm{g})$ & $14 \cdot 6$ & 0.27 & 14.7 & 0.24 & $15 \cdot 5$ & 0.27 & 0.04 \\
\hline Polyunsaturated fat (g) & $12 \cdot 0$ & 0.16 & $12 \cdot 2$ & 0.16 & $12 \cdot 3$ & 0.18 & 0.36 \\
\hline Trans fat $(\mathrm{g})$ & $0 \cdot 11^{*}$ & 0.01 & 0.13 & 0.01 & 0.15 & 0.01 & 0.003 \\
\hline Total fat (\% of energy) & $28 \cdot 3^{\star}$ & 0.30 & $29 \cdot 1^{*}$ & 0.29 & $30 \cdot 3$ & 0.32 & $<0.0001$ \\
\hline Dietary cholesterol (mg) & $102^{*}$ & $4 \cdot 74$ & $102^{*}$ & $4 \cdot 22$ & 122 & $4 \cdot 74$ & 0.001 \\
\hline Dietary fibre $(\mathrm{g})$ & $20 \cdot 8^{\star}$ & 0.28 & $21 \cdot 1^{\star}$ & 0.26 & $19 \cdot 8$ & 0.30 & 0.02 \\
\hline n-3 Fatty acids (mg) & 0.14 & 0.01 & 0.14 & 0.01 & 0.13 & 0.01 & 0.78 \\
\hline n-6 Fatty acids (mg) & 0.34 & 0.01 & 0.37 & 0.01 & 0.39 & 0.01 & 0.04 \\
\hline Glycaemic index & $41 \cdot 2^{*}$ & 0.36 & $39 \cdot 8$ & 0.34 & $39 \cdot 4$ & 0.39 & 0.001 \\
\hline Glycaemic load & $93 \cdot 1^{*}$ & $1 \cdot 12$ & $89 \cdot 1^{*}$ & 1.06 & 85.4 & $1 \cdot 20$ & $<0.0001$ \\
\hline
\end{tabular}

MASALA, Mediators of Atherosclerosis in South Asians Living in America.

*Mean value was significantly different from that of the third tertile of length of residence in the USA: $P<0.05$.

†Variables are reported as mean and standard error, adjusted for age, sex and energy intake, except for energy intake itself. Differences between groups were examined using generalized linear models using Dunnett's adjustment for multiple comparisons. 
Table 3 Daily micronutrient intakes of the MASALA study participants according to length of residence in the USA†

\begin{tabular}{|c|c|c|c|c|c|c|c|}
\hline \multirow[b]{3}{*}{ Nutrient (/d) } & \multicolumn{6}{|c|}{ Length of residence in the USA } & \multirow[b]{3}{*}{$P$ for treno } \\
\hline & \multicolumn{2}{|c|}{ 1st tertile $(n 298)$} & \multicolumn{2}{|c|}{ 2nd tertile ( $n$ 293) } & \multicolumn{2}{|c|}{ 3rd tertile $(n 283)$} & \\
\hline & Mean & SE & Mean & SE & Mean & SE & \\
\hline Vitamin C (mg) & 217 & 3.94 & $218^{*}$ & 3.77 & 208 & 4.23 & 0.07 \\
\hline Thiamin (mg) & 1.48 & 0.02 & 1.48 & 0.02 & 1.44 & 0.02 & 0.10 \\
\hline Riboflavin (mg) & 1.63 & 0.02 & 1.62 & 0.02 & 1.58 & 0.02 & 0.08 \\
\hline Vitamin $B_{6}(\mathrm{mg})$ & 1.90 & 0.02 & 1.91 & 0.02 & 1.87 & 0.02 & $0 \cdot 10$ \\
\hline Vitamin $B_{12}(\mathrm{mg})$ & $2 \cdot 41$ & 0.08 & 2.43 & 0.08 & 2.57 & 0.08 & 0.34 \\
\hline Folate $(\mu \mathrm{g})$ & $416^{*}$ & 5.41 & $410^{*}$ & $4 \cdot 81$ & 388 & $5 \cdot 41$ & $<0.0001$ \\
\hline Vitamin A (RE) & 1765 & $47 \cdot 2$ & 1862 & $45 \cdot 0$ & 1778 & $50 \cdot 6$ & 0.77 \\
\hline Vitamin D $(\mu \mathrm{g})$ & $2 \cdot 17$ & 0.12 & $2 \cdot 14$ & 0.12 & 2.01 & 0.13 & 0.91 \\
\hline Vitamin E (mg) & $5 \cdot 66$ & 0.09 & $5 \cdot 73^{\star}$ & 0.08 & 5.47 & 0.09 & 0.07 \\
\hline $\mathrm{K}(\mathrm{mg})$ & $3664^{*}$ & 35.9 & $3690^{*}$ & 34.3 & 3554 & 38.5 & 0.01 \\
\hline $\mathrm{Na}(\mathrm{mg})$ & 2733 & $32 \cdot 9$ & 2753 & 31.5 & 2690 & 35.4 & 0.36 \\
\hline $\mathrm{Ca}(\mathrm{mg})$ & 944 & 19.9 & 927 & 9.0 & 884 & 21.4 & 0.08 \\
\hline
\end{tabular}

MASALA, Mediators of Atherosclerosis in South Asians Living in America; RE, retinol equivalents.

*Mean value was significantly different from that of the third tertile of length of residence in the USA: $P<0.05$.

†Variables are reported as mean and standard error, adjusted for age, sex and energy intake. Differences between groups were examined using generalized linear models using Dunnett's adjustment for multiple comparisons.

Table 4 Weekly intakes of selected foods and food groups of the MASALA study participants according to length of residence in the USA $\dagger$

\begin{tabular}{|c|c|c|c|c|c|c|c|}
\hline \multirow[b]{3}{*}{ Food/food group (servings/week) } & \multicolumn{6}{|c|}{ Length of residence in the USA } & \multirow[b]{3}{*}{$P$ value } \\
\hline & \multicolumn{2}{|c|}{ 1st tertile ( $n$ 298) } & \multicolumn{2}{|c|}{ 2nd tertile ( $n$ 293) } & \multicolumn{2}{|c|}{ 3rd tertile (n 283) } & \\
\hline & Median & $\begin{array}{l}\text { 25th-75th } \\
\text { percentiles }\end{array}$ & Median & $\begin{array}{l}\text { 25th-75th } \\
\text { percentiles }\end{array}$ & Median & $\begin{array}{l}\text { 25th-75th } \\
\text { percentiles }\end{array}$ & \\
\hline Alcoholic beverages & $0.00^{*}$ & $0.00-1.00$ & $0.00^{*}$ & $0.00-1.50$ & 0.25 & $0 \cdot 00-3 \cdot 25$ & $<0.001$ \\
\hline Beans and lentils & $7 \cdot 13^{*}$ & $4 \cdot 38-10 \cdot 5$ & $6 \cdot 50^{*}$ & $3 \cdot 88-10 \cdot 5$ & $5 \cdot 63$ & $3.00-8.50$ & 0.0001 \\
\hline Breads, cereals and grains & $18 \cdot 3^{\star}$ & $13 \cdot 0-24 \cdot 4$ & $18 \cdot 0$ & $12 \cdot 0-23 \cdot 8$ & $16 \cdot 3$ & $11 \cdot 9-21 \cdot 8$ & 0.01 \\
\hline Fats and oil & $0.57^{*}$ & $0.00-4.00$ & $1 \cdot 25$ & $0.00-5.00$ & 2.00 & $0.00-5.00$ & 0.02 \\
\hline \multicolumn{8}{|l|}{ Fried snack foods } \\
\hline All & $0.75^{\star}$ & $0.25-2.00$ & 0.75 & $0.25-2 \cdot 50$ & 0.63 & $0.00-1.63$ & 0.07 \\
\hline South Asian-style & 1.50 & $0.50-3.50$ & 1.50 & $0.50-3.25$ & $1 \cdot 25$ & $0.50-3.25$ & 0.38 \\
\hline Western-style & $0 \cdot 25^{\star}$ & $0.00-0.50$ & 0.13 & $0.00-0.38$ & 0.00 & $0.00-0.38$ & 0.06 \\
\hline Fruits & $13 \cdot 8$ & $9 \cdot 00-20 \cdot 8$ & 14.5 & $9 \cdot 50-21 \cdot 8$ & 14.9 & $8 \cdot 50-23 \cdot 3$ & 0.62 \\
\hline Meat, poultry, seafood and eggs & 0.25 & $0.00-2.00$ & 0.25 & $0.00-1.50$ & 0.50 & $0.00-1.50$ & 0.78 \\
\hline Milk and dairy & $24 \cdot 9^{*}$ & $16 \cdot 0-33 \cdot 5$ & 22.9 & $15 \cdot 3-31 \cdot 8$ & $22 \cdot 0$ & $12 \cdot 5-31 \cdot 8$ & 0.05 \\
\hline Mixed dishes & $0.63^{\star}$ & $0 \cdot 25-1 \cdot 13$ & 0.75 & $0 \cdot 25-1 \cdot 25$ & 0.75 & $0 \cdot 25-1 \cdot 38$ & 0.04 \\
\hline Nuts & $5 \cdot 44^{*}$ & $2 \cdot 00-8 \cdot 38$ & $6 \cdot 00$ & $3 \cdot 00-10 \cdot 5$ & $7 \cdot 00$ & $3 \cdot 00-10 \cdot 0$ & 0.06 \\
\hline Rice and rice preparations & $3.50^{*}$ & $1.63-7.00$ & $2 \cdot 25^{\star}$ & $1 \cdot 00-4 \cdot 25$ & 2.00 & $0.75-4.00$ & $<0.0001$ \\
\hline Sugar, candy and jam & $7 \cdot 25^{\star}$ & $0.75-15 \cdot 5$ & $7 \cdot 00^{*}$ & $1 \cdot 00-14.8$ & $4 \cdot 13$ & $0.50-10 \cdot 8$ & 0.01 \\
\hline Sugar-sweetened beverages & 0.00 & $0.00-0.50$ & 0.00 & $0.00-0.50$ & 0.00 & $0.00-0.25$ & 0.17 \\
\hline \multicolumn{8}{|l|}{ Sweets } \\
\hline All & $2 \cdot 00$ & $0.75-4 \cdot 25$ & $1 \cdot 88$ & $0.75-4 \cdot 25$ & $1 \cdot 88$ & $0.63-3.83$ & 0.54 \\
\hline South Asian-style & 0.50 & $0.00-1.50$ & 0.38 & $0.00-1.63$ & 0.25 & $0 \cdot 00-1 \cdot 25$ & 0.17 \\
\hline Western-style & $1 \cdot 13$ & $0.50-2.25$ & 1.00 & $0.25-2 \cdot 50$ & $1 \cdot 12$ & $0.25-2 \cdot 63$ & 0.86 \\
\hline \multicolumn{8}{|l|}{ Vegetables } \\
\hline All & $29 \cdot 0^{*}$ & $21 \cdot 5-38 \cdot 8$ & $28 \cdot 0$ & $20 \cdot 8-39.8$ & $26 \cdot 1$ & $19 \cdot 3-38 \cdot 0$ & 0.10 \\
\hline Starchy & $1.50^{\star}$ & $1.00-3.00$ & $1.50^{\star}$ & $0.75-4.25$ & 1.00 & $0.50-3.00$ & 0.002 \\
\hline Other & $26 \cdot 8$ & $20 \cdot 0-35 \cdot 6$ & $26 \cdot 3$ & $18 \cdot 6-37 \cdot 3$ & 24.5 & $17 \cdot 3-36 \cdot 0$ & 0.15 \\
\hline
\end{tabular}

MASALA, Mediators of Atherosclerosis in South Asians Living in America.

${ }^{*}$ Median value was significantly different from that of the third tertile of length of residence in the USA: $P<0.05$

†Data on food group intake are presented as median value with interquartile range. Differences between groups were examined using the Kruskal-Wallis test.

lentils, breads, cereals and grains, rice and rice preparations, as well as starchy vegetables and sugar, candy and jam.

Few studies have examined whether nutrient and food intakes differ by length of residence in the USA among South Asians. In a study of seventy-three South Asian immigrants living in New York City and Washington DC, differences in nutrient and food intakes after moving to the USA by length of residence in the USA were examined ${ }^{(20)}$. Regardless of the length of stay in the USA, the participants reported consuming more fruit juice, snack chips, fruits, margarine, cola and alcoholic beverages after moving to the USA; and in contrast to recent residents, participants living in the USA for 10 years or longer reported lower 
intakes of foods such as ghee (clarified butter), milk and other dairy products. In the MASALA study, we did not query specifically whether participants had changed their dietary patterns after moving to the USA; however, intakes of alcoholic beverages and butter (including ghee), margarine and oil were higher among South Asians in the highest tertile of length of residence in the USA as compared with the lowest and middle tertiles. More recently, Lesser and colleagues ${ }^{(21)}$ examined the associations between acculturation and dietary patterns among 207 participants of South Asian origin belonging to the MultiCultural Community Health Assessment Trial. Length of residence in Canada (examined as quartiles with cut-off values: $\leq 13.8$ years, $13.9-21.1$ years, $21.2-32.1$ years and $\geq 32.2$ years) was associated with positive dietary behaviours and practices including increased fruit and vegetable consumption and a preference for cooking methods such as grilling as compared with deep-frying. However, a longer period of residence in Canada was also associated with less healthy practices including higher intakes of meat and sugar-sweetened beverages and higher frequencies of eating outside the home, indicating mixed results from time spent in Canada. Findings from the MASALA study are consistent with those reported for South Asian immigrants residing in Europe ${ }^{(22,23)}$, suggesting that immigration, culture and food environment all influence dietary intake among this population subgroup.

Our study has several strengths. South Asians are a growing minority in the USA with extensive disparities for chronic diseases such as type 2 diabetes and heart disease; however, few, if any studies have examined the role of factors such as diet and acculturation on these increased risks. The MASALA study has carefully collected and detailed information on several traditional and novel risk factors using validated methods. Dietary assessment in the cohort was conducted using a culturally appropriate FFQ whose relative validity has been previously examined. However, in general, diet assessment is fraught with measurement error which can sometimes be differential, and most commonly includes under-reporting of energy, nutrients and foods, thereby affecting the results of dietdisease relationships ${ }^{(24,25)}$. In addition, the study was observational in design and residual confounding due to factors that were imprecisely or not measured is also a possibility. The MASALA cohort is largely representative of the middle-aged and older South Asian population currently residing in the USA. However, the high educational and socio-economic attainment and older age of the cohort may limit the generalizability of any findings to those with lower education levels and younger ages.

The key finding of the current study was that dietary intakes among South Asian immigrants living in the USA differed by the length of residence in the USA, with largely mixed benefits. For example, a longer length of residence in the USA was associated with lower intakes of foods such as rice, starchy vegetables, and sugar, candy and jam but was positively associated with intakes of fats and oils. For some nutrients and foods (e.g. carbohydrate and alcohol), differences across groups were statistically significant; however, the absolute differences were relatively small. While small differences in intakes of nutrients and foods may not always translate to significant associations with chronic disease risk, they may be indicative of an overall eating pattern which may be more strongly correlated with health outcomes and therefore critical in informing policy recommendations. For example, health promotion programmes for South Asian immigrants should focus on emphasizing healthy behaviours such as the consumption of traditional foods like beans and lentils while limiting intakes of simple carbohydrates, fats and oil.

South Asians in the USA are a fast-growing ethnic minority and exhibit a higher risk for chronic diseases, thereby potentially posing a significant health-care expense $^{(1,2)}$. Dietary behaviours are an important predictor of chronic disease risk ${ }^{(6,26)}$. Factors such as length of stay in the USA play an important role in the diet and health of immigrant populations ${ }^{(27-29)}$. Therefore, understanding the changing dietary intakes of immigrant groups is crucial for developing effective diet and lifestyle interventions aimed at reducing the chronic disease risk of diverse populations.

\section{Acknowledgements}

Financial support: The MASALA study was supported by the National Institutes of Health (grant number 1R01-HL-093009). Data collection at UCSF was also supported by NIH/NCRR UCSF-CTSI (grant number UL1 RR024131). A.M.K. was also supported by grant K24HL112827. The funders had no role in the design, analysis or writing of this article. Conflict of interest: None. Authorship: N.R.K. and A.M.K. contributed to the original design and to data collection for the MASALA study. S.A.T. was responsible for the design and analysis of this report and drafted the manuscript. N.R.K., A.M.K., M.D.G. and D.D. consulted on the analysis. All authors made critical comments during the preparation of the manuscript. S.A.T. has primary responsibility for the final content. All authors read and approved the final version of the paper. Ethics of buman subject participation: The study was conducted according to the guidelines laid down in the Declaration of Helsinki and all procedures involving human subjects were approved by the institutional review boards of UCSF and NWU. All study participants signed informed consent. Further, the institutional review board of Johns Hopkins Bloomberg School of Public Health deemed this secondary analysis plan using de-identified data as not constituting human subjects research. 


\section{References}

1. US Census Bureau (2012) The Asian Population: 2010. https://www.census.gov/prod/cen2010/briefs/c2010br-11.pdf (accessed February 2015).

2. Palaniappan LP, Araneta MR, Assimes TL et al. (2010) Call to action: cardiovascular disease in Asian Americans: a science advisory from the American Heart Association. Circulation 122, $1242-1252$.

3. Shah A \& Kanaya AM (2014) Diabetes and associated complications in the South Asian population. Curr Cardiol Rep 16, 476.

4. Shah AD, Vittinghoff E, Kandula NR et al. (2015) Correlates of prediabetes and type II diabetes in US South Asians: findings from the Mediators of Atherosclerosis in South Asians Living in America (MASALA) study. Ann Epidemiol 25, 77-83.

5. Page RL (2006) Acculturation in Mexican immigrants: a concept analysis. J Holistic Nurs 24, 270-278.

6. Satia-Abouta J, Patterson RE, Neuhouser ML et al. (2002) Dietary acculturation: applications to nutrition research and dietetics. J Am Diet Assoc 102, 1105-1118.

7. Bermudez OI, Falcon LM \& Tucker KL (2000) Intake and food sources of macronutrients among older Hispanic adults: association with ethnicity, acculturation, and length of residence in the United States. J Am Diet Assoc 100, 665-673.

8. Murtaugh MA, Herrick JS, Sweeney C et al. (2007) Diet composition and risk of overweight and obesity in women living in the southwestern United States. J Am Diet Assoc 107, 1311-1321.

9. Gregory-Mercado KY, Staten LK, Ranger-Moore J et al. (2006) Fruit and vegetable consumption of older Mexican-American women is associated with their acculturation level. Ethn Dis 16, 89-95.

10. Neuhouser ML, Thompson B, Coronado GD et al. (2004) Higher fat intake and lower fruit and vegetables intakes are associated with greater acculturation among Mexicans living in Washington State. J Am Diet Assoc 104, 51-57.

11. Gordon-Larsen P, Harris KM, Ward DS et al. (2003) Acculturation and overweight-related behaviors among Hispanic immigrants to the US: the National Longitudinal Study of Adolescent Health. Soc Sci Med 57, 2023-2034.

12. Batis C, Hernandez-Barrera L, Barquera S et al. (2011) Food acculturation drives dietary differences among Mexicans, Mexican Americans, and Non-Hispanic Whites. J Nutr 141, 1898-1906.

13. van Rompay MI, McKeown NM, Castaneda-Sceppa C et al. (2012) Acculturation and sociocultural influences on dietary intake and health status among Puerto Rican adults in Massachusetts. J Acad Nutr Diet 112, 64-74.

14. Romero-Gwynne E, Gwynn D, Grivetti L et al. (1993) Dietary acculturation among Latinos of Mexican descent. Nutr Today 28, 6-12.
15. Holmboe-Ottesen G \& Wandel M (2012) Changes in dietary habits after migration and consequences for health: a focus on South Asians in Europe. Food Nutr Res 2012, 56.

16. Kanaya AM, Kandula N, Herrington D et al. (2013) Mediators of Atherosclerosis in South Asians Living in America (MASALA) study: objectives, methods, and cohort description. Clin Cardiol 36, 713-720.

17. Kelemen LE, Anand SS, Vuksan V et al. (2003) Development and evaluation of cultural food frequency questionnaires for South Asians, Chinese, and Europeans in North America. I Am Diet Assoc 103, 1178-1184.

18. Merchant AT, Anand SS, Kelemen LE et al. (2007) Carbohydrate intake and HDL in a multiethnic population. Am J Clin Nutr 85, 225-230.

19. Merchant AT, Anand SS, Vuksan V et al. (2005) Protein intake is inversely associated with abdominal obesity in a multi-ethnic population. J Nutr 135, 1196-1201.

20. Raj S, Ganganna P \& Bowering J (1999) Dietary habits of Asian Indians in relation to length of residence in the United States. J Am Diet Assoc 99, 1106-1108.

21. Lesser IA, Gasevic D \& Lear SA (2014) The association between acculturation and dietary patterns of South Asian immigrants. PLoS One 9, e88495.

22. Wandel M, Raberg M, Kumar B et al. (2008) Changes in food habits after migration among South Asians settled in Oslo: the effect of demographic, socio-economic and integration factors. Appetite 50, 376-385.

23. Patel JV, Vyas A, Cruickshank JK et al. (2006) Impact of migration on coronary heart disease risk factors: comparison of Gujaratis in Britain and their contemporaries in villages of origin in India. Atherosclerosis 185, 297-306.

24. Kipnis V, Subar AF, Midthune D et al. (2003) Structure of dietary measurement error: results of the OPEN biomarker study. Am J Epidemiol 158, 14-21.

25. Trabulsi J \& Schoeller DA (2001) Evaluation of dietary assessment instruments against doubly labeled water, a biomarker of habitual energy intake. Am J Physiol Endocrinol Metab 281, E891-E899.

26. World Health Organization (2003) Diet, Nutrition and the Prevention of Chronic Diseases. Joint WHO/FAO Expert Consultation. WHO Technical Report Series no. 916. Geneva: WHO.

27. Koya DL \& Egede LE (2007) Association between length of residence and cardiovascular disease risk factors among an ethnically diverse group of United States immigrants. J Gen Intern Med 22, 841-846.

28. Pan Y-L, Dixon Z, Himburg S et al. (1999) Asian students change their eating patterns after living in the United States. J Am Diet Assoc 99, 54-57.

29. Lee S-K, Sobal J \& Frongillo EA (1999) Acculturation and dietary practices among Korean Americans. J Am Diet Assoc 99, 1084-1089. 
Appendix

Food groups and average serving sizes in the MASALA study analysis

Food group
Alcoholic beverages
Beans and legumes
Breads, cereals and grains

Fats and oil

Fruit

Meat, poultry, seafood and eggs

Milk and dairy

Mixed dishes

Nuts and oilseeds

Rice and rice preparations

Snack foods

South Asian-type

Western-type

Sweets

South Asian-type

Western-type

Sugar, candy and jam

Sugar-sweetened beverages

Vegetables

Starchy

Other
Food item and average serving size

Beer (average serving: 1 bottle or $355 \mathrm{ml}$ ), wine (average serving: $5 \mathrm{fl}$ oz or $150 \mathrm{ml}$ ), spirits (average serving: $1.5 \mathrm{fl} \mathrm{oz}$ or $45 \mathrm{ml}$ )

All cooked dried beans and lentils (average serving: $1 / 2$ cup or $125 \mathrm{ml}$ )

Breads (average serving: 1 slice), ready-to-eat and cooked breakfast cereals (average serving: 1 cup or $250 \mathrm{ml}$ ), muffins (average serving: 1 small), crackers (average serving: 2 pieces), traditional preparations such as roti (flatbread), chapatti (flatbread), puri (fried flatbread), mathri (fried flatbread), naan (leavened flatbread) and paratha (flatbread) (average serving: one, 6" $(15 \mathrm{~cm})$ diameter; or $4^{\prime \prime}(10 \mathrm{~cm})$ diameter for puri)

Butter, margarine, ghee (clarified butter) and oil used for tempering or on boiled rice and breads (average serving: 1 tsp or 1 pat)

Citrus and non-citrus fruits (average serving, depending on type of fruit: 1 medium fruit or 1 wedge or $1 / 2$ cup) and $100 \%$ fruit juices (average serving: $3 / 4$ cup or $175 \mathrm{ml}$ )

Preparations with beef, pork, goat or lamb (average serving: $1 / 2$ cup or $125 \mathrm{ml}$ if curry; $6^{\prime \prime}(15 \mathrm{~cm}) \mathrm{kebab}$ or 2 kofta or $3^{\prime \prime}(7.5 \mathrm{~cm})$ patty if hamburger or minced meat type; medium if roast or steak), hot dogs and sausages (average serving: 1 hot dog or 2 links), lunch meat (average serving: $1 \mathrm{slice}$ or $30 \mathrm{~g}$ ), liver (average serving: medium), preparations with chicken (average serving: 4 pieces if fried wings; $1 / 2$ cup or $125 \mathrm{ml}$ if curry; medium if roast such as tandoori or tikka), preparations with fish and seafood (average serving: medium if steamed or baked; $1 / 2$ cup or $125 \mathrm{ml}$ if curry; $1 / 2$ can or $50 \mathrm{~g}$ if canned; 5 fish sticks if deep-fried), preparations with egg (average serving: 1 egg)

Milk (average serving: 1 cup or $250 \mathrm{ml}$ or 2 tbsp or $30 \mathrm{ml}$ in tea or coffee), cream and half and half (average serving: 1 tbsp or $15 \mathrm{ml}$ ), cheese (average serving: 1 slice), cream cheese (average serving: 2 tbsp), yoghurt (average serving: $3 / 4$ cup or $175 \mathrm{ml}$ ) and cottage cheese or paneer (average serving: $1 / 2$ cup or $125 \mathrm{ml})$

Soup (average serving: $1 / 2$ cup or $125 \mathrm{ml}$ ), pizza (average serving: 1 medium slice) and pasta (average serving: 1 cup)

Coconut (average serving: 1 tbsp or $1^{\prime \prime}(2.5 \mathrm{~cm})$ piece), nuts (average serving: 2 tbsp) and peanut butter (average serving: 1 tbsp or $15 \mathrm{ml}$ )

Rice: plain boiled, fried rice and pulaos (similar to pilaf) (average serving: 1 cup)

Samosas (generally fried pastry with filling) (average serving: 1 medium), fritters or pakoras (average serving: $1 / 2$ cup), chaat (savoury snack) (average serving: $1 / 2$ cup), bhajia, sev and gathia (types of snack foods made with chickpea flour) (average serving: 1/4 cup), tikias (potato patties and vegetable cutlets) (average serving: one, $3^{\prime \prime}(7.5 \mathrm{~cm})$ diameter)

French fried potatoes (average serving: 1 cup), popcorn, potato chips and nachos (average serving: $1 / 2$ cup)

Traditional South-Asian sweets and desserts including barfi (sweet confectionery made with condensed milk and sugar), ladoo (mostly flour-based dessert), halwa (dense pudding), kheer (pudding), gulab jamun (milk solids-based dessert) and rasgullas (cottage cheese-based dessert) (average serving: 1 piece or $1 / 2$ cup or $125 \mathrm{ml}$ )

Cake (average serving: 1 slice or $\left.2^{\prime \prime} \times 4^{\prime \prime} \times 1^{\prime \prime}(5 \mathrm{~cm} \times 10 \mathrm{~cm} \times 2.5 \mathrm{~cm})\right)$, doughnuts and sweet rolls (average serving: 1 piece), pies and tarts (average serving: 1 slice or 1/6th pie) and cookies (average serving: 1 cookie)

Sugar or honey (average serving: 1 tsp or 1 packet), chocolate (average serving: 1 small bar or $45 \mathrm{~g}$ ), candy (average serving: 2 pieces) and jam (average serving: $1 \mathrm{tsp})$

Fruit drinks (average serving: 1 cup or $250 \mathrm{ml}$ ) and non-diet soft drinks (average serving: 1 can or $355 \mathrm{ml}$ )

Potato and potato preparations (average serving: 1 medium or 1 cup for boiled, mashed or baked; $1 / 2$ cup or $125 \mathrm{ml}$ for all other) and green peas (average serving: $1 / 2$ cup)

Cauliflower, cabbage, green peppers, broccoli, okra, dark green leafy vegetables, tomato, onion, squash, pumpkin, aubergine, lettuce, cucumber, carrots, sweet potato, leeks (average serving: $1 / 2$ cup or $125 \mathrm{ml}$ )

MASALA, Mediators of Atherosclerosis in South Asians Living in America. 